\title{
LA PERCEPCIÓN DE LOS ESTUDIANTES UNIVERSITARIOS EN LA MEDIDA DE LA COMPETENCIA DOCENTE: VALIDACIÓN DE UNA ESCALA
}

\author{
Raziel Acevedo Álvarez \\ Profesor de la Universidad de Costa Rica \\ María José Fernández Díaz \\ Profesora de la Universidad Complutense de Madrid
}

Recibido 20-II-2004 • Aceptado 13-IV-2004

\begin{abstract}
Resumen: El propósito de este estudio consiste en desarrollar y validar la medida de un constructo de competencia docente universitaria, utilizando un cuestionario de opinión de los estudiantes, con 30 items. En la muestra han participado 374 estudiantes de tres departamentos de la Universidad de Costa Rica, Sede de Guanacaste, cuyo mayor grupo está representado por estudiantes de primer ingreso (39,4\%), con una edad media de 19,9 años. Para validar el constructo propuesto se han aplicado los modelos de ecuaciones estructurales a seis factores de competencia docente universitaria, identificados como: organización, evaluación, presentación, entusiasmo, interacción y valoración global del estudiante. Las universidades interesadas en la innovación pueden utilizar estos factores para desarrollar planes de formación y mejora de sus docentes.
\end{abstract}

Palabras clave: Evaluación, Profesores Universitarios, Instrumentos de Evaluación, Análisis Factorial Confirmatorio, AMOS, EQS, Competencia Docente.

\begin{abstract}
The purpose of this study is to develop and to validate a construct of a university teaching competence, using the students' rating with 30 items. 374 students of the three departments at the University of Costa Rica, Guanacaste Campus have participated. The largest group of participants is represented by junior students (39.4\%), with an average of 19.9 years old. To valid the present, construct we have applied the models of the structural equation to six university teacher competences, such as organization, evaluation, presentation, enthusiasm, interaction and the student's global valuation. The universities interested in the innovation can use these factors to develop formation plans to upgrade their professors.
\end{abstract}

Key words: Educational Evaluation, Evaluation Instruments, Confirmatory Factor Analysis, AMOS, Teacher Competence.

\section{Parte: El punto de partida \\ 1. Las líneas de investigación sobre el tema}

La evaluación del profesorado universitario es, posiblemente, uno de los temas sobre educación superior que mayor número de debates, literatura e investigación ha generado en el campo de la evaluación universitaria. Su amplio desarrollo se puede justificar y caracterizar fundamentalmente, porque la competencia docente es un componente básico en el proceso de enseñanzaaprendizaje y, en consecuencia, podría decirse que la calidad de la universidad y su reputación, se sustenta en la calidad de sus profesores. No obstante, este pensamiento ha responsabilizado al docente del logro o no de la calidad educativa, olvidando que ello depende de muchos componentes para su éxito, en donde indudablemente el profesorado es una parte.

En la literatura más significativa sobre el tema, se encuentran dos líneas de investigación mayoritarias: 1) aquellos que tratan de identificar los factores de eficacia docente y 2) los que estudian la relación existente entre los resultados de estas evaluaciones con diferentes variables o con factores de sesgo (consultar Craton y Smith, 
1990; March, 1997, 2000; Cohen, 1986; Aleamori, 1999; Abrami y otros, 1997; McKeachie, 1990; Feldman, 1997).

Los estudios relacionados con el sesgo, representados entre otros por Centra (1993), Aleamori (1999), Feldman (1997), Marsh y Roche (1997) y Fernández (1998), estudian las características de la personalidad o del comportamiento del profesor que puedan verse afectadas, por elementos externos a la docencia universitaria, como: el tamaño de la clase, la obligatoriedad de la materia, el sexo del estudiante o del profesor y la disciplina académica, entre otras. Dentro de esta misma línea, los trabajos de Prosser y Trigwell (1990) y Gibbs (1992) consideran que el interés de estas evaluaciones se ha ido incrementando hacia las características de los estudiantes (hábitos de estudio; motivación hacia el aprendizaje, entre otros). Otros investigadores por su parte, Ramsden y Martín (1992) y Murray (1997), se ocupan por conocer la utilidad de estas evaluaciones como un componente de juicio en la toma decisiones sobre la contratación y ascenso de los profesores.

La segunda línea de estudios, es de mayor aceptación y desarrollo, se ha dedicado a buscar dimensiones o factores que caractericen al "buen profesor" y que puedan ser utilizados como predictores de evaluación docente universitaria. Acerca de este tema es posible encontrar en la literatura una cantidad considerable de propuestas de diferentes contextos, entre las que destacan Marsh (1984), Centra (1990), Abrami (1990) y Feldman (1976a y 1976b), y otros.

De todas las propuestas analizadas, nosotros consideramos que las características del docente universitario se pueden agrupar, sin afán reduccionista, en relación con ciertos elementos comunes referidos a: organización, evaluación de la clase, comunicación e interacción con los estudiantes. García Ramos (1997), destaca una cierta coincidencia en el nombre de esas dimensiones y en sus definiciones, llamadas a veces con nombres iguales o con nombres diferentes pero con el mismo significado. También Marsh $(1997,2000)$ ha demostrado la existencia de cierto consenso y añade que tienen un carácter multidimensional, son fiables y válidas.

En todo este contexto, observamos cierta coherencia entre los investigadores sobre varias dimensiones afines en los estudios y de acuerdo con nuestro pensamiento, esa coincidencia surge en las dimensiones relacionadas con organización, interacción, comunicación, presentación y evaluación. Estas dimensiones, son comúnmente utilizadas como criterios de medida en los diferentes instrumentos sobre la evaluación de la competencia docente universitaria y señalan el camino seguido por la investigación sobre el tema.

No obstante, aunque esta relación entre investigadores es notoria, nos plantea varias inquietudes: ¿cuáles dimensiones son las predictoras de competencia docente? ¿Cuántas se deben emplear para obtener una visión general sobre la acción docente universitaria? Refiriéndose a lo anterior Abrami, d'Apollonia y Cohen (1990) determinan que los predictores utilizados para la medida de la competencia docente, varían dependiendo de las particularidades que estén siendo objeto de estudio. En este sentido, cada autor los define de acuerdo con sus objetivos o con las necesidades institucionales predominantes en ese momento. Por esa razón, no encontramos investigaciones referidas a las dimensiones más importantes y cuantas son necesarias para construir un instrumento de medida de la competencia docente.

En un análisis de los instrumentos encontrados en la voluminosa literatura sobre este campo de investigación, Marsh y Roche (1997) llegan a la conclusión de que pocas de estas investigaciones han sido evaluadas profundamente con la dedicación necesaria en términos de potencial, sesgo y validez. El autor encontró en muchos de 
estos instrumentos, insuficiente estructura teórica y un limitado análisis estadístico. Una afirmación de esta naturaleza nos regresa al punto de partida y nos lleva a plantear otras interrogantes, ¿son válidas las evaluaciones que se aplican a los docentes universitarios? ¿Han sido minuciosamente analizadas sus propiedades psicométricas relacionadas con fiabilidad y validez? En este punto hemos de resaltar que la mayoría de las quejas presentadas por los profesores universitarios evaluados, tienen relación directa más con su uso y aplicación indebidos, que con los problemas de validez.

\section{Dimensiones y predictores identificados por los estudiantes asociados con la competencia docente}

La búsqueda por las dimensiones o predictores asociados con la competencia docente, desde la visión de los alumnos, ha sido la fuente de trabajo de numerosos estudios e investigaciones, apoyados en diferentes posturas teóricas las cuales les han permitido identificar los predictores de evaluación de la acción universitaria.

En esta incesante búsqueda se pueden observar trabajos muy importantes como los de Feldman (1976a, 1976b, 1987, 1989) que destaca la existencia de 14 a 20 dimensiones analizadas por los investigadores. En su estudio el autor esquematiza estas investigaciones e identifica varios constructos $^{1}$, agrupados concisamente en tres factores de primer orden y relacionados con el rol del profesor: 1) el presentador (un hombre actor o presentador, presentador de materiales), 2) facilitador del aprendizaje (interactúa o recíproco) y 3) regulador del aprendizaje (director o manager).

Otro trabajo muy difundido en la literatura es el de Marsh (1982, 1984), Marsh y Dunkin (1992) y algunos colaboradores. En él se propone como variable dependiente la valoración global del estudiante y de variables independientes nueve factores que son: 1) aprendizaje, 2) entusiasmo, 3) organización, 4) interacción con el grupo, 5) apoyo individual, 6) amplitud y cobertura, 7) justicia en los exámenes, 8) asignaciones y 9) dificultad del curso. Para consolidar y demostrar la solidez de los nueve factores, Marsh y Hocevar (1991) realizan un análisis factorial confirmatorio al $\mathrm{SEEQ}^{2}$ y concluyen en la definición de un constructo que utiliza los nueve factores.

Hemos de destacar que la estructura del SEEQ ha sido considerada y estudiada durante muchos años por un gran número de investigadores y evaluadores en diferentes países del mundo como Estados Unidos, Australia, Hong Kong y España ${ }^{3}$. Marsh y todos sus colaboradores han creado un cuerpo de literatura muy importante que ha conferido un potente impulso al estudio de la evaluación de la docencia universitaria.

Respecto a los últimos años observamos trabajos como el de Patrick y Smart (1998) donde se identifican tres dimensiones: la habilidad para cambiar al estudiante, organización y presentación. Esta última dimensión está incluida en el estudio de Kwan (1999), aplicado en la Universidad de Hong Kong, en donde se recolectaron 5000 evaluaciones de profesores en 75 departamentos, empleando un instrumento que además de la dimensión organización y presentación, contenía cinco más relacionadas principalmente con: resultado de aprendizaje, motivación, feedback, interacción y ayuda individual.

La interacción del profesor con los estudiantes también está contenida en un instrumento de Ballantyne, Bothwick, y Packer (2000), quienes proponen siete dimensiones para el estudio: organización y preparación de la clase, presentación de la clase, participación de los estudiantes en la clase, aprendizaje de los estudiantes, evaluación de los estudiantes de su aprendizaje, interacción del profesor y los estudiantes, métodos/áreas de enseñanza y retroalimentación en la enseñanza. 
Por medio del análisis factorial, Swartz y otros (1990) identifican dos factores con la eficacia docente: (1) claridad instruccional de presentación, y (2) administración de la conducta de los estudiantes. Lowman y Mathie (1993) también insisten en la utilización de dos factores : (1) intelectualmente estimulante, y (2) relaciones con los estudiantes. No obstante, en otros estudios se identifican más y diferentes factores de eficacia docente. Por ejemplo, Brown y Watkins (1994) consideran tres factores (1) afectividad, (2) sistematización y (3) estimulación. El mismo número de factores son descritos por Rindermann (2001) los cuales son: estructuración, la competencia docente y entusiasmo. Otros investigadores han sugerido más factores como los siete de Ramsden (1991) o los nueve que destaca Marsh y Dunkin (1992).

De acuerdo con nuestra opinión, los autores mantienen su coincidencia en los factores relacionados con organización, evaluación, interacción, trato con los estudiantes y entusiasmo. Estos continúan siendo los más difundidos y utilizados como criterios de evaluación docente desde la perspectiva de los estudiantes.

En el contexto español, también se observan múltiples trabajos sobre el tema, como los de Alvarez y García (1999), Escudero (1999), Jornet (1988) y Cajide (1994), entre otros.

Entre los diversas propuestas existentes, Tejedor (1990) insiste en siete dimensiones o factores, altamente correlacionadas entre sí, que deben ser empleadas para evaluar al docente: (a) cumplimiento del profesor, (b) calidad y desarrollo del programa, (c) dominio de la asignatura, (d) interacción con los alumnos, (e) recursos utilizados y prácticas, (f) exámenes y (g) valoración global del profesor. A su vez González Such y otros (1999), destacan tipologías de calidad docente como: cumplimiento de obligaciones, conocimiento de la materia, desarrollo de la clase, materiales y programa, actitud del profesor y evaluación.

Un instrumento muy importante de estudiar y de mencionar, por la profundidad en el análisis de sus componentes y por la rigurosidad con que se valida el constructo competencia docente a través del análisis factorial confirmatorio, es el CEDA 4 de García Ramos (1996, 1997, 1997b, 1998). El CEDA enumera seis dimensiones implicadas en la evaluación de la competencia del docente universitario, que se describen como: 1) Programa-organización de la enseñanza, 2) dominio de contenidos-claridad expositiva, 3) motivación de aprendizaje-incremento del interés del alumno, 4) interacción con el grupo de clase, 5) atención individual al alumno, 6) evaluación-exámenes. Para englobar las seis dimensiones mencionadas, el CEDA se divide en tres partes: valoración de la materia, valoración analítica del docente y valoración global del mismo.

Últimamente la búsqueda de las dimensiones para la evaluación de la competencia docente universitaria continúa siendo objeto de estudio y se pueden observar algunos ejemplos destacados en la siguiente tabla.

Nosotros consideramos que de las diferentes propuestas analizadas, muchas de esas dimensiones pueden llegar a ser sintetizadas alrededor de organización, interacción con los estudiantes, preparación, habilidades para comunicar y estimular a los estudiantes y justicia en la nota. En nuestra opinión, las primeras dos dimensiones aparecen con mayor frecuencia en los estudios; tanto en el ámbito internacional como en el español (Marsh, 2000; Ting, 2001; García Ramos, 1997; González Such, 1999; y Tejedor, 1990). 
Tabla 1

Dimensiones de finales de los noventas y al 2001

\begin{tabular}{|c|c|c|c|c|c|}
\hline $\begin{array}{c}\text { Marsh } \\
(2001)\end{array}$ & $\begin{array}{l}\text { Ting } \\
(\mathbf{2 0 0 1})\end{array}$ & $\begin{array}{l}\text { Young } \\
\text { (1999) }\end{array}$ & $\begin{array}{c}\text { Kolitch y } \\
\text { Dean (1999) }\end{array}$ & $\begin{array}{c}\text { Ridermann y } \\
\text { Amelang (2001) }\end{array}$ & $\begin{array}{c}\text { Jackson } \\
\text { y otros (1999) }\end{array}$ \\
\hline $\begin{array}{l}\text { 1- Aprendizaje } \\
\text { y valor. } \\
\text { 2- Interacción } \\
\text { con el grupo. } \\
\text { 3- Raport } \\
\text { individual. } \\
\text { 4- Exámenes } \\
\text { calificaciones. } \\
\text { 5- Carga de tra- } \\
\text { bajo dificultad. } \\
\text { 6- Organización } \\
\text { y claridad. } \\
\text { 7- Entusiasmo. } \\
\text { 8- Amplitud } \\
\text { de enfoque. } \\
\text { 9- Trabajo } \\
\text { extra clase. }\end{array}$ & $\begin{array}{l}\text { 1- Diseño } \\
\text { del curso. } \\
\text { 2- Rendimiento } \\
\text { del profesor. } \\
\text { 3- Evaluación } \\
\text { global del } \\
\text { diseño, el } \\
\text { rendimiento } \\
\text { y el esfuerzo } \\
\text { dedicado al } \\
\text { curso. }\end{array}$ & $\begin{array}{l}\text { 1- Entusiasmo. } \\
\text { 2- Conocimiento } \\
\text { de la materia. } \\
\text { 3- Habilidad } \\
\text { para integrar } \\
\text { la información. } \\
\text { 4- Habilidad } \\
\text { para estimular } \\
\text { el pensamiento. }\end{array}$ & $\begin{array}{l}\text { 1- Comunicación. } \\
\text { 2- Planteamiento } \\
\text { y organización. } \\
\text { 3- Manejo } \\
\text { de clase. }\end{array}$ & $\begin{array}{l}\text { 1- Estructura. } \\
\text { 2- Competencia } \\
\text { docente. } \\
\text { 3- Entusiasmo. }\end{array}$ & $\begin{array}{l}\text { 1- Apoyo a los } \\
\text { estudiantes. } \\
\text { 2- Valor } \\
\text { del curso. } \\
\text { 3- Organización } \\
\text { y diseño. } \\
\text { 4- Justicia en } \\
\text { la calificación. } \\
\text { 5- Cantidad } \\
\text { de trabajo. }\end{array}$ \\
\hline
\end{tabular}

\section{Dimensiones conceptuales de competencia docente universitaria}

La competencia docente es el criterio que con mayor frecuencia se utiliza para evaluar al profesorado universitario y desde una perspectiva general, podría decirse es "aquel que demuestra eficacia en el logro de los objetivos que son propios de su trabajo a nivel universitario -docencia, investigación y servicios a la comunidad" (De Miguel, 1998, 72). En otras palabras, lo anterior se refiere según Schalock (1993) a la "preparación específica del docente universitario para cumplir de manera adecuada con las responsabilidades" (p. 67) y las funciones profesionales.
En este sentido, se busca destacar los atributos característicos que un docente debe tener para una práctica segura de su profesión. Tales atributos han de ser una composición interrelacionada a la cultura, el comportamiento y la acción, entre otros. Cuando los profesores logran reunir e integrar dichos componentes, adquieren ciertas competencias que le sirven para enfrentar la enseñanza universitaria con una mejor visión.

Según Alvarez (1999) la definición de la competencia docente es un problema muy complejo e involucra una serie de factores internos y externos del profesor. No obstante, ello es el resultado de la interacción entre cuatro macro-variables: 1 ) oficio docente, 2) el arte de ser profesor, 3) las 
condiciones estructurales y 4) los dilemas y paradojas. Este estudio se fundamenta a partir de la primera macro-variable.

El oficio docente es un conjunto de saberes y concepciones sobre las funciones a desarrollar por el profesor que dan como consecuencia una forma eficaz de enseñar: profesionalidad (dominio de los contenidos, dedicación, preparación de las clases, asunción de roles, innovación docente) conocimiento y características de las necesidades del alumnado (necesidad de acogida, necesidad de motivación, carencias formativas, necesidad de estructuración de estudio), destrezas docentes (claridad expositiva, claridad en los criterios de evaluación, aplicabilidad del contenido de la enseñanza, dominio del proceso de argumentación y demostración, adquisición y utilización de recursos didácticos personales, habilidad para la comunicación con los alumnos, adaptación de la enseñanza). (Alvarez, 1999, 278).

Considerando la propuesta del autor y analizando los estudios sobre la percepción de los estudiantes de sus profesores universitarios, podemos manifestar que los profesores competentes destacan en las evaluaciones en las dimensiones relacionadas con el oficio docente, y que se refieren a: organización, conocimiento de la materia, comunicación, interacción con los estudiantes, entusiasmo y métodos de evaluación.

\subsection{Organización y conocimiento de la materia}

La organización de la materia de clase, es una de las dimensiones más utilizadas en la literatura sobre el tema. $\mathrm{Su}$ empleo es común en los diferentes instrumentos propuestos por investigadores, evaluadores y administradores universitarios. Los estudios más prestigiosos de Marsh (2001), Patrick (1998) y Kwan (1999) proponen la utilización de dicho criterio, como uno de los predictores más destacados de competencia docente.
Un profesor organizado es considerado como aquel que planifica con anterioridad su clase, busca, ordena los recursos, organiza profundamente todos los componentes, llámese objetivos, actividades, textos y exposiciones, entre otros. García (1997) y Marsh (1987) han demostrado en sus estudios empíricos que esta dimensión tiene una influencia muy fuerte sobre otros criterios de la competencia docente universitaria.

Además, un profesor ha de conocer profundamente lo que enseña, ha de ser un especialista en constante búsqueda de conocimiento, pero se hace necesario combinar los contenidos que involucra su materia, para generar resultados positivos de aprendizaje. Bien es conocido que el conocimiento de la materia no es garantía de éxito; no es suficiente ser un especialista al más alto nivel, es más importante contar con las habilidades que permitan una forma de intercambio con los estudiantes, para que las experiencias de clase tengan algún significado para ellos.

En este sentido, García y Congosto (2000), aseveran que el dominio de contenidos de una asignatura o disciplina es la condición necesaria. Pero, la condición suficiente para que se produzca una acción educativa-formadora eficaz, esta "ligada al resto de procesos que facilitan la comunicación de los contenidos y la motivación de aprendizaje que generan en el estudiante. Ambas condiciones son básicas" (García y Congosto, 2000, p. 245).

Podemos manifestar que el dominio de la materia se encuentra muy relacionado con la organización y la planificación de la clase misma. Trent y Cohen (1973), Marsh (2001), De Miguel (1998), Nuhfer (1996), Frey, Leonard y Beatty (1975), Feldman (1997), Marsh y Roche (1993, 1997), Coffey y Gibbs (2001) y Centra (1993), entre otros, han propuesto el empleo de esta dimensión como un factor de eficacia docente.

En los meta-análisis más importantes sobre el tema Cohen $(1986,1987)$ con un grupo de 41 estudios bien escogidos, el 
autor encontró un coeficiente de 0,34 entre el rendimiento del estudiante y el conocimiento de la materia, poniendo en evidencia una moderada relación entre ambas variables. No obstante, en el mismo estudio, el autor resalta que se observa una alta correlación, coeficiente de 0,57, entre la estructura y logro, lo cual está altamente relacionado con la preparación y organización de la clase. Los resultados evidencian el peso de esta dimensión en la evaluación de la docencia universitaria.

\subsection{Comunicación con los estudiantes}

Los profesores deben ser capaces de comunicar sus ideas efectivamente en diferentes formas y también, a diferentes personas. En esta línea, Baugh (1983) y Taylor (1988), expresan que el aprendizaje se facilita cuando el profesor y los estudiantes comparten por medio de la comunicación, significados de ideas o sentimientos con el uso de sonidos convencionales, signos o gestos, apoyados en el empleo de símbolos escritos que son mutuamente entendidos.

La comunicación puede ser aún más efectiva cuando tiene significados culturales que son compartidos por todas las personas en la clase. Esto significa, teóricamente, que dos culturas pueden tener el mismo lenguaje, pero variedad de grados de comprensión de los disímiles significados de las palabras; éstas se pueden escribir igual, pero generalmente tienen diferentes significados que dependen del lugar, el acento, la intensidad y muchos otros elementos más. Collins (1988) y Heath (1983), tratando sobre la comunicación en el aula, asumen que los profesores necesitan reconocer esta realidad, para hacer ajustes que le permitan facilitar el aprendizaje a sus estudiantes.

En una revisión sobre el tema De la Orden (1990) encontró que la habilidad del profesor para implicar eficazmente a los estudiantes en tareas de aprendizaje es un predictor importante de eficacia, pues existen un gran número de estudios correlacionales que lo indican.

\subsection{Interacción y entusiasmo}

Un docente universitario ha de ser capaz de interactuar con los estudiantes, de manera que ayude al crecimiento académico y personal de éstos. Los estudiantes, realizan un mejor aprendizaje en situaciones donde se sienten cómodos y, de alguna forma, apoyados por los docentes. Cuando los alumnos perciben que existe cierta afinidad y familiaridad, están más atentos y comprometidos con los procesos de clase. Jackson (1999) declara que el estudiante observa la habilidad del profesor para apoyar a los estudiantes, creando una atmósfera que conduzca al aprendizaje.

Con un proceso adecuado de interacción lo estudiantes se sienten libres de expresar su opinión, apoyarse en conversaciones entre ellos, y sobre todo, animan con ello el desarrollo de la clase

Aunque, no podemos creer que los estudiantes quietos, ordenados o atentos se sientan bien dentro de la clase. Los profesores necesitan ser sensibles a las necesidades emocionales de sus estudiantes y responder a sus relaciones interpersonales, buscando diferentes formas de interrelacionarse con ellos, de manera que les permita conocer una parte de sus intereses, necesidades, metas y preocupaciones. Las relaciones interpersonales acercan al profesor a la realidad del estudiante y abren las puertas a la comprensión, la sensibilidad y sobre todo a la humanización del proceso de enseñanza.

Los profesores exitosos tienden a ser los que pueden utilizar un rango de las estrategias de enseñanza y un rango de estilos de interacción, presentándose ante sus estudiantes entusiasmados por los diferentes elementos de la clase. Estos son capaces de aceptar críticas, agilizar los 
temas difíciles, se muestran atentos y contentos ante sus estudiantes. En fin, estos aspectos se encuentran constantemente en las investigaciones sobre eficacia docente, lo que sugiere según Doyle (1985) que los profesores eficaces ajustan su enseñanza para conocer las necesidades de diversos estudiantes y las demandas que ellos tienen sobre metas, temas y métodos educacionales.

Good (1983) destaca que el uso de diversas estrategias ocurre en el contexto de la "enseñanza activa" que es determinada y diagnóstica, que responde tanto a las necesidades de los estudiantes como del currículo. En este sentido, la interacción juega un papel determinante, ya evidenciado en los estudios de Cohen (1986) y Marsh (2001) quienes encontraron una correlación de 0,52 para la interacción del profesor con el estudiante.

\subsection{La evaluación de la enseñanza}

Los profesores han de promocionar el rendimiento y el conocimiento del estudiante universitario a un nivel más complejo. Foster (1991) asevera que el planeamiento y la evaluación de la enseñanza permiten al docente observar si el estudiante comprende la materia de clase y la relaciona con destrezas que le permiten ascender a niveles más elevados: ésta es la esencia de la docencia y el aprendizaje. El nivel de habilidad, conocimiento y de destrezas, ha de ser evaluado a intervalos regulares para determinar el progreso de los estudiantes y también, para estudiar otras acciones que le permitan al estudiante superar las áreas o los capítulos más problemáticos de la clase.

No obstante, para lograr una importante relación entre los intereses docentes y estudiantiles Jackson (1999) afirma que debe existir justicia a la hora de valorar a los estudiantes. Los criterios utilizados tienen que ser los mismos para todos estudiantes sin ninguna distinción que les permita la duda. Los docentes se deben presentar imparciales en sus ponderaciones sobre el rendimiento de los estudiantes. Además, las pruebas han de mantenerse relacionadas con el contenido estudiado en clase y con el tiempo de estudio de los estudiantes.

La dimensión evaluación es muy señalada por los investigadores sobre el tema y es uno de los predictores destacados por los autores más relevantes. García (1997) propone que esta dimensión influye directamente sobre otras dimensiones de evaluación docente.

Los autores mencionan que los factores mencionados de evaluación del profesor pueden variar de acuerdo al tamaño de la clase, como en el caso de apoyo e interacción. En un meta-análisis muy importante Cohen (1987) reportó los siguientes coeficientes: estructura $(0,55)$, interacción $(, 52)$, habilidades $(, 50)$, global del curso $(, 49)$, total del instructor $(, 45)$, aprendizaje (,39), apoyo (,32), evaluación (,30), feedback $(, 28)$; interés /motivación $(, 15)$ y dificultad $(-, 04)$, claridad de los objetivos del curso $(0,35)$, habilidades $(0,56)$, conocimiento sobre la materia $(, 34)$ y sensibilidad con el nivel de clase y progreso $(0,30)$. Se observa una alta correlación entre estructura y logro pero tiene relación con la preparación y organización (,57). Los hallazgos de este autor son muy consistentes para el uso de tales dimensiones en la evaluación de estudiantes de sus profesores universitarios.

Hasta aquí, se han señalado las dimensiones más representativas de la competencia docente universitaria, reunidas alrededor de: organización, interacción con los estudiantes, preparación, habilidades para comunicar y estimular a los estudiantes y justicia en la nota. Los criterios observados nos brindan una visión general de competencia docente y pueden ser utilizados como esquemas de evaluación del profesorado universitario. 


\section{Parte: Propuesta empírica}

\section{Problema y objetivos}

Los problemas de investigación de este estudio se fundamentan en el análisis de la literatura sobre la evaluación de la competencia docente. Esta profundización permite plantearse ciertas interrogantes a resolver ¿Cuáles dimensiones son las predictoras de competencia docente? ¿Cuántas se deben emplear para obtener una visión general sobre la acción del docente universitario? Con el fin de empezar a dar respuesta a estos problemas, se definen básicamente los siguientes objetivos generales:

- Identificar las dimensiones o factores utilizados como criterios de medida de la competencia docente universitaria a partir de la percepción de los estudiantes.

- Estudiar y adaptar una escala de medida de la competencia docente universitaria.

- Analizar las propiedades psicométricas de la escala propuesta.

- Validar un constructo relacionado con la competencia docente universitaria.

\section{Método}

\subsection{Muestra del estudio}

Esta formada por 374 estudiantes de la Universidad de Costa Rica, Sede de Guanacaste, pertenecientes a tres departamentos: Artes, Filosofía y Letras, Ciencias
Sociales y Ciencias Básicas. La media de edad es 19,9 años, un $49 \%$ son mujeres y el $50 \%$ hombres, mayoritariamente de primer año (57\%). La selección se ha realizado por medio del procedimiento de muestreo aleatorio simple, entre todos los matriculados del año 2001.

\subsection{Instrumento: el cuestionario}

De acuerdo con este estudio, la competencia docente universitaria ha sido observada en relación con dos criterios. El primero, se desprende de la revisión de la literatura especificada en la primera parte de nuestra exposición. El segundo, toma en cuenta la naturaleza y el contenido del instrumento facilitado por el Centro de Evaluación Académica de la Universidad de Costa Rica. A nuestro juicio, podemos observar la calidad de la enseñanza basado en varias dimensiones como: planificación general, métodos y recursos, exposición, amplitud, métodos evaluativos, interacción, apoyo, entusiasmo y la valoración global del profesor y del curso. Las dimensiones empleadas en la construcción de este instrumento han sido identificadas por los investigadores y por el gran cuerpo de literatura que tiene el tema en debate. El detalle de tales elementos se puede observar en la tabla №2

Observada la tabla se pueden resumir brevemente las características del instrumento:

- Cuestionario global: 34 ítems, con dos ítems de control, o criterios, comunes en este tipo de instrumentos (ver anexo №1). 
Tabla 2

Núcleo teórico, dimensiones e indicadores

\begin{tabular}{|c|c|c|}
\hline Estructura & Subdimensión & Indicadores \\
\hline \multirow{6}{*}{ 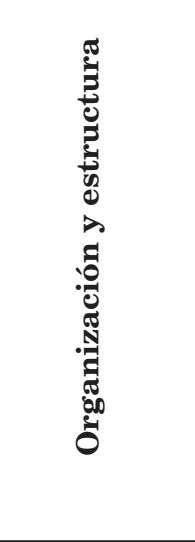 } & Planificación general del curso & Preparación y organización de las clases \\
\hline & Mítodos y recursos empleados & Utilización de métodos y recursos de instrucción \\
\hline & Exposición, claridad de contenidos & Dominio de los temas - Claridad y orden \\
\hline & Amplitud & $\begin{array}{l}\text { Contexto a sus ideas y conceptos } \\
\text { Tratamiento a los temas }\end{array}$ \\
\hline & Mítodo evaluativo & $\begin{array}{l}\text { Usticia e imparcialidad a la hora de evaluar } \\
\text { y calificar. Alaci ón y definición de los criterios } \\
\text { de evaluación. Feedback de la evaluación }\end{array}$ \\
\hline & Valor del aprendizaje & $\begin{array}{l}\text { Valor del curso (profesional, humano, dificultad, } \\
\text { cantidad de trabajo). Expectativa e interés }\end{array}$ \\
\hline \multirow{3}{*}{ 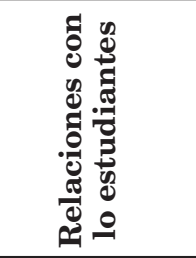 } & Interacción & $\begin{array}{l}\text { Estimula y provoca la participación, el di! logo, la } \\
\text { reflexión y debate }\end{array}$ \\
\hline & Apoyo & $\begin{array}{l}\text { Escucha dudas y críticas } \\
\text { Aoya a los estudiantes }\end{array}$ \\
\hline & Entusiasmo & $\begin{array}{l}\text { etitud activa y din ! mica } \\
\text { Entusiasmo e interés mostrado en clase }\end{array}$ \\
\hline 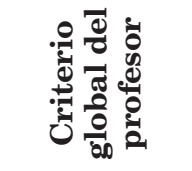 & Valoración global & General con el profesor y el curso. \\
\hline
\end{tabular}

- Operacionalización de las dimensiones: a) Planificación general del curso y de la clase: se calificarán tomando en cuenta aspectos relacionados con la organización general del curso y la clase, la definición previa de sus objetivos clases y la planificación general e individual de la clase. b) Utilización de métodos y recursos de instrucción: mide una variedad de recursos de instrucción como la utilización de la multimedia, de lecturas apropiadas a cada tema, de vídeos, diapositivas y trasparencias. También considera técnicas pedagógicas referentes a: demostraciones, clase magistral y trabajo en grupo. c) Dominio de los temas: esta dimensión involucra el dominio de los contenidos de clase, el orden de exposición de los mismos y la claridad expositiva. d) Amplitud: observa el tratamiento que brinda a los temas, la contextualización brindada a sus ideas, conceptos y al manejo de los contenidos temáticos. e) Método evaluativo: mide la justicia e imparcialidad a la hora de evaluar y calificar al estudiante, la definición de los criterios de evaluación y su relación con 
los temas vistos en clase y al tipo de feedback que genera con la evaluación del curso. f) Interacción: valúa si el docente estimula y provoca la participación, el diálogo, la reflexión y el debate en clase, escucha las dudas y las críticas, si mantiene buenas relaciones con los estudiantes. g) Apoyo: mide concretamente la disponibilidad en la atención de los estudiantes antes y después de clase. h) Entusiasmo: evalúa si mantiene una actitud activa y dinámica clase, si se manifiesta entusiasmado al impartir sus lecciones, evidenciando con ello que se siente a gusto en ella. i) Valoración global: estos criterios finales resumen la opinión general de los estudiantes sobre el profesor y el curso.

- $\quad$ Escala aditiva global tipo Lickert.

- Puntuación de los ítems: escala de cinco puntos, uno es considerado el menor y cinco mayor.

- Formulación de los ítems en sentido positivo.

- Asimilación de dimensiones para uso formativo. En sentido sumativo, únicamente el referente de la escala global.

\subsection{Análisis y resultados}

Iniciamos analizando la fiabilidad del instrumento de medida. Para estimar esta característica psicométrica se emplea el paquete estadístico SPSS versión 10.1. En cuanto a la fiabilidad de este cuestionario, una vez que se eliminaron los ítems 29, 30, 31 y 32 , por su poco ajuste, se obtuvo un coeficiente alfa de Cronbach de 0,94. Este valor es altamente satisfactorio y destaca la consistencia interna del instrumento. Posteriormente se divide la muestra entre los dos departamentos con mayor cantidad de sujetos, y se alcanzan índices similares al de la muestra total (0,93 para Ciencias Sociales y 0,94 para Ciencias Básicas). Los resultados indican consistencia en el juicio de los estudiantes, demostrando que estos diferencian muy bien a unos profesores de otros. Al respecto Overall y Marsh (1980) destacan que la fiabilidad en las encuestas de estudiantes se entiende como el acuerdo relativo (unanimidad) entre valoraciones de diferentes estudiantes dentro de la misma clase, bajo la asunción de que cualquier varianza específica del estudiante individual es aleatoria y debería ser considerada como varianza de error.

Analizado y estimado el coeficiente de fiabilidad, decidimos comprobar su otra propiedad psicométrica: la validez. Este aspecto resulta un poco más complicado de estudiar, porque para ello se ha de tener en cuenta la integración de múltiples juicios. No obstante, Cronbach (1954) considera que la validez más importante es la de constructo, porque en ella se reúnen todos los tipos de validez existentes.

\subsection{Análisis factorial exploratorio}

Para iniciar, se trata de reducir la información por medio del análisis factorial exploratorio, utilizando el método de componentes principales con rotación varimax. Primeramente, ha de asegurarse que la matriz de correlaciones sea significativa, por medio de: a) la prueba de esfericidad de Bartlett (ji- cuadrado $=4534.201, \mathrm{p}=$ 0 '000, con $435 \mathrm{gl}$ ) indica que no es una matriz de identidad debido a la existencia de inter-correlaciones significativas, $\mathrm{y}$ b) K.M.O = 0,94, esto demuestra que el conjunto de variables colectivas alcanzan el umbral necesario de suficiencia muestral. Ambos índices justifican plenamente la aplicación del análisis factorial. Seguidamente, se analizan las comunalidades, que se encuentran dentro de los rangos considerados normales. Luego, al proceder con la selección de los factores se obtienen seis que explican el $61,17 \%$ de la varianza 
y cuyo primer factor representa el 40,56\%. Para contar con una mejor visión de los factores extraídos, son rotados, por medio del método varimax, alcanzado una estructura con seis elementos denominados como: entusiasmo, interacción, evaluación, organización-recursos, presentación-dominio y valoración global.

\subsection{Análisis factorial confirmatorio}

\subsubsection{Especificación e identificación}

Determinados los factores, se procede con la validación de constructo utilizando los modelos de ecuaciones estructurales, hipotetizando que la evaluación global del estudiante es directamente influenciada por el entusiasmo y la interacción del profesor. Estas a su vez son variables endógenas afectadas directamente por la evaluación y la presentación. La organización, variable exógena, influye directa e indirectamente sobre todas las variables anteriores. El modelo recursivo puede verse en el gráfico № 1 .

Se observa una variable exógena y cinco endógenas, que se manifiestan por 30 variables observadas que se ordenan con su correspondiente variable latente de la siguiente forma (ver anexo № 1): entusiasmo (ítems $\mathrm{n}^{\circ}: 25,20,21,23,27$ ), interacción

Gráfico $\mathrm{N}^{0} 1$

Modelo porpuesto

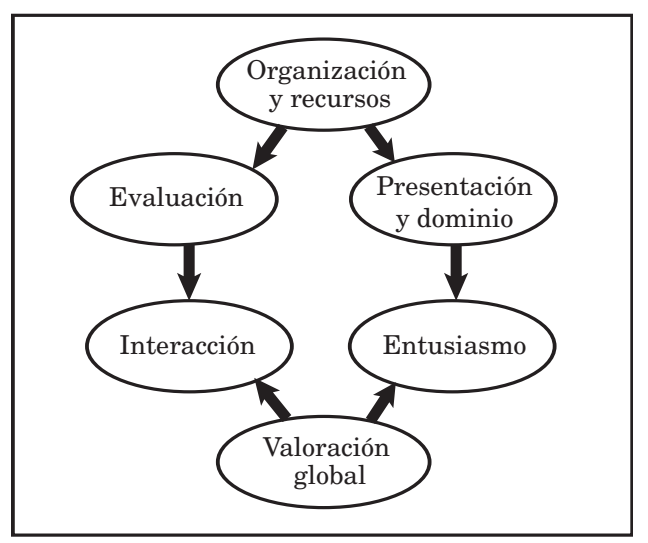

(ítems $\left.\mathrm{n}^{\circ}: 22,24,26,28,19\right)$, evaluación (ítems $\left.\mathrm{n}^{\circ}: 2,5,6,17,11,15\right)$, organización y recursos (ítems $\mathrm{n}^{\circ}: 1,9,4,3,10,13$ ), presentación y dominio (ítems $\mathrm{n}^{\circ}: 12,7,14,18,16$, 8) y valoración global (ítems $\left.n^{\circ}: 33,34\right)$. Para proceder con la estimación del modelo, las variables observadas número $1,2,16,19,25$ y 33 son ajustadas a uno. En este modelo se proponen 137 parámetros de estimación.

\subsubsection{Estimación y evaluación}

La estimación y evaluación del modelo de medida, tanto de la variable exógena como de las endógenas, se realiza utilizando el paquete EQS 6.0. El primer modelo de referencia es el de medida de la variable exógena. Su análisis demuestra que las variables observadas miden bien la variable latente, pues sus valores son todos significativos. Además, al observar los índices de ajuste los valores son todos satisfactorio (NFI 0,87, CFI 0,92, MFI 0,65, GFI 0,88, AGFI 0,86, CMI/Df=2,55, RMR 0,048, RMSEA 0,059). El segundo modelo de medida, las variables endógenas, aporta resultados similares al modelo anterior $\mathrm{y}$ se deduce que su ajuste es satisfactorio (NFI 0,92, CFI 0,95, MFI 0,98, GFI 0,97, AGFI 0,95, CMI/Df=2,31, RMR 0,046, RMSEA 0,065). Cabe mencionar que el c2 de bondad del ajuste es significativo en ambos modelos de medida, por tanto se podría concluir que los modelos no ajustan completamente a los datos. No obstante, este tema ha sido muy debatido debido a la falta de normalidad multivariante, por esa razón, la gran mayoría de autores recomiendan eliminar este criterio y observar otras medidas de ajuste adicionales para complementar el juicio que se haga sobre un modelo determinado.

La medida más empleada en lugar del c2 es el CMI/df, que según Green y otros (1997) el valor adecuado ha de ser inferior a tres. En nuestro modelo de estudio obtenemos un 2,41 , por tanto se juzga satisfactorio. 
Considerado el ajuste de los modelos de medida (exógeno y endógeno), se procede con la estimación y evaluación del modelo estructural propiamente dicho, empleando el paquete estadístico AMOS 4,0. La estimación realizada por este programa informático se puede observar en el gráfico № 2 .
Un análisis detallado de cada uno de los parámetros estimados (pesos de las regresiones, sus estimados, interceptos y varianzas) permite manifestar que el peso y la influencia de unas variables sobre otras es positiva y muy significativa, lo que demuestra una estructura consistente. Respecto de 
Analizada la información sobre el modelo estructural, procedemos a evaluar las medidas de ajuste. Hemos de tomar en cuenta que únicamente se reporta información sobre las medidas más empleadas por los investigadores.

Medidas de ajuste absoluto: Estas medidas determinan el grado en que el modelo propuesto (estructural y de medida) predicen la matriz de correlación o covarianza observada. Evidentemente, éstas no hacen distinción entre si el modelo de ajuste es mejor o peor. La primera medida obtenida es ji- cuadrado (C2 1010,70 con 399 grados de libertad) decidimos eliminarlo del análisis y utilizar las propuestas alternativas, como el FMIN y F0.

En el modelo que proponemos estos índices tienen un valor de 2,71 el primero y 1,64, el segundo. Los coeficientes demuestran valores de ajuste bastante aceptables, porque se encuentran por debajo del límite normal $(3,0)$.

Ahora bien, al observar el RMSEA cuyo índice es 0,064 , con un " $p$ " $=0,00$, se demuestra que el error es bueno y evidencia un buen ajuste del modelo de estudio propuesto.

Medidas de ajuste incremental: estas medidas valoran el ajuste incremental del modelo comparado con un modelo nulo, que se supone como un modelo de un único factor sin error de medida. En este caso, el valor del chi-cuadrado del modelo nulo o alternativo es $\mathrm{C} 2=40625,733$ con 465 grados de libertad y un nivel de significación del 0,00, con un CMIN/df = 87367. Estos valores son muchísimo más altos que los del modelo que estamos proponiendo. Bentle y Bonett (1980), proponen que, si balanceamos este valor con el obtenido por nuestro modelo, podemos considerar que el nuestro no es tan deficiente.

A parte de esta recomendación, un tanto subjetiva, se puede tomar en cuenta el índice de Tucker y Lewis (TLI), el índice de ajuste normado (NFI) y el índice de ajuste comparado (CFI). Según Hu y Bentler
(1999) estos deben permanecer por encima de 0,95 , para ser considerados como un excelente ajuste.

En el modelo de estudio los índices TLI es 0,98, el NFI es 0,97 y el CFI 0,98, estos son valores muy altos y ofrecen una medida válida de ajuste. Analizando estos coeficientes, observamos que todos exceden los niveles recomendados para el ajuste incremental, o sea mayor de 0,90, lo que aporta argumentos adicionales para calificar de aceptable el modelo que estamos proponiendo.

Medidas de ajuste de la parsimonia: esta última medida, según Hair y otros (1999), evalúa la parsimonia del modelo propuesto, mediante la valoración del ajuste del modelo, frente al número de coeficientes estimados necesarios para lograr ese nivel de ajuste.

En este sentido, se pueden observar varias medidas muy relacionadas entre sí, las cuales son apropiadas para evaluar directamente el modelo que proponemos y cuyos valores han de superar el mínimo de significación $(0,90)$.

En nuestro modelo el PNFI o índice de ajuste parsimonioso normado y el índice de ajuste parsimonioso comparado o PCFI, es de 0,83 el primero y 0,84 el segundo, por tanto, se debe aceptar con precaución esta medida porque es marginal al valor recomendado por los autores. Pese a ello, estas medidas permanecen dentro de los rangos normales.

Otro índice teórico que también ofrece información relevante para determinar el ajuste del modelo es el valor de Hoelter. Según el autor el valor ha de ser mayor a 200. En el modelo que proponemos obtenemos 165, con significación 0,05 , y 173 para 0,01 ; podemos ver que estos valores también son marginales y han de ser tomados con precaución por estar debajo del valor señalado. Al respecto podemos tomar una aclaración de Arbuckle (1997) quién no se fía del límite en 200 y da por buenos todos rangos que se obtengan alrededor de 170 . 
Dentro de estos índices también se recomienda observar el ji-cuadrado normado (CMI/Df), cuyos umbrales señalados están entre 1 y 5 . El CMI/Df del modelo propuesto es 2,41 , lo cual es un valor que se encuentra dentro de los rangos recomendados por los autores como una buena estimación.

Se puede manifestar, en una observación final, que los valores obtenidos en el ajuste del modelo de estudio son más que aceptables, por tanto, es posible considerarlo una propuesta válida para explicar la percepción de los estudiantes respecto a la competencia docente universitaria. Se ha de destacar, que se obtuvieron resultados muy similares a los anteriores cuando la muestra se dividió en los dos departamentos mayoritarios.

\section{Conclusiones}

De acuerdo con lo expuesto anteriormente, podemos señalar:

1) La evaluación de la competencia docente es un tema de debate permanente en el contexto de las instituciones de educación superior, debido principalmente al pensamiento que señala a los profesores como responsables, entre otras cosas, de introducir cambios y matices para modular el clima del aula, la calidad del trabajo y la orientación de los aprendizajes de los estudiantes. Eso significa que en las actitudes y aptitudes de los docentes, descansa gran parte del éxito y el prestigio de las universidades. Claro está, ello supone una enorme responsabilidad que no es única de los profesores, sino, de todos los componentes de la institución universitaria, llámese: administración, biblioteca, servicios, entre otros.

2) Consideramos que evaluar la actividad docente universitaria es y será un problema permanente, esencialmente por dos razones no solucionadas: a) la teoría no ha determinado hasta dónde el rendimiento del estudiante se debe a su contexto, interés o al docente, b) Es difícil aislar todos los componentes de la docencia, por tanto, las diferentes propuestas de evaluación serán siempre incompletas.

3) Si bien es cierto que se han observado ciertas dimensiones afines utilizadas en diferentes estudios y que se refieren a: organización, interacción, comunicación y evaluación, entre otras, no hay un acuerdo sustantivo entre cuántas o cuáles utilizar, dado que depende de los objetivos del evaluador y de los intereses de la institución.

4) Por otra parte, los resultados obtenidos en la estimación y evaluación del modelo de estudio propuesto por los autores, son más que aceptables pues superan en algunos casos los valores establecidos. La información aportada por la estimación del modelo en ambos programas (EQS. AMOS), nos parece apropiada y sumamente valiosa porque cumple alta y moderadamente con la recomendaciones de ajuste; hecho que indica empíricamente la validez del constructo definido aquí como la percepción de la competencia docente universitaria por parte de los estudiantes. Lo cual es una explicación plausible del fenómeno estudiado, aunque esto no elimina la existencia de modelos alternativos igualmente válidos.

5) Por tanto, tal y como se deduce del modelo propuesto en nuestro estudio, un profesor valorado como competente por sus estudiantes, es aquél que se presenta ante sus estudiantes con clases muy organizadas, con una 
estructura lógica que les permite entender la secuencia de los temas y comprender la materia de estudio. Es claro en sus explicaciones e ideas, conoce la materia, se muestra activo y dinámico durante el desarrollo de la clase. Además, trata de permanecer cercano al estudiante, escuchando amablemente sus dudas, respondiendo y atendiendo los problemas de estos. También es justo a la hora de calificarlos, tratando de antemano de no quitar o dar más puntos al estudiante, sin una justificación objetiva. Para ello define previamente los objetivos que se desea conseguir en la evaluación, es claro en sus criterios e introduce únicamente aquellos temas vistos en clase. Ante todo es un ser humano, preparado, comprensivo, comunicativo y organizado.

6) Es evidente que utilizar un solo criterio de eficacia docente no es suficiente para evaluar la competencia docente universitaria. Somos concientes de la complejidad que envuelve la docencia universitaria y aunque la evaluación de estudiantes es fiable y válida, sus criterios no pueden ser los únicos para conocer realmente la actividad.

7) El instrumento propuesto por los investigadores es fiable y válido, y puede ayudarnos a mostrar un fragmento de la visión que los estudiantes tienen del profesor, mediante unos factores que proveen una base comprensiva para su evaluación. Esto indica la posibilidad de promediar holísticamente la competencia docente. Sin embargo, se debe tener en cuenta que los resultados obtenidos en este estudio pertenecen a una muestra de un contexto determinado, aunque ello no invalida los resultados, sí se recomienda mayor investigación adicional, en otros contextos y con otras muestras, que ayuden a determinar, las dimensiones necesarias o mínimas para elaborar un instrumento de esta naturaleza y validar el constructo.

Finalmente, los factores utilizados en este estudio (organización, evaluación, presentación, entusiasmo e interacción) pueden ser utilizados en las Universidades que estén interesadas en la innovación y la mejora, a fin de implementar planes de formación que ayuden a los docentes a desarrollar y perfeccionar estas competencias tan necesarias, en una profesión complicada como es la docencia universitaria.

\section{Notas}

1. Los constructos son: la simulación del interés del profesor, el conocimiento de la materia, habilidad para hablar, naturaleza y valor de los materiales del curso, expansión intelectual del profesor, estimulación del interés, entusiasmo, transmisión intelectual, preparación y organización, claridad y entendimiento, habilidad de elocución, sensibilidad hacia el progreso de la clase, claridad de objetivos, valor de los materiales del curso, justicia e imparcialidad, dirección de clase, feedback de los estudiantes, discusión de clase, cambio intelectual, respeto a los estudiantes, Disponibilidad y ayuda, dificultad y cantidad de trabajo.

2. SEEQ siglas en inglés de Student Evaluation of Educational Quality.

3. Marsh, 1983, 1984, 1987, 1991; Marsh y otros, 1985; Marsh y Bailey, 1993; Marsh y Dunkin, 1992; Marsh y Roche, 1992, 1994, 1997, 2000; Marsh y Hocevar, 1991, 1984; Marsh y otros, 1997; Marsh; Touron y Wheeler (1985).

4. CEDA significa, Cuestionario de Evaluación Docente por el Alumnado.

5. $\quad$ Ver Hair pág. 646. 


\section{Referencias bibliográficas}

Abrami, P.C.; D'apollonia, S. \& Cohen, P. "Validity of Student Ratings of Instruction: What We Know and What We Do Not". En Journal of Educational Psychology, 82 (2), pp.219231. 1990.

Abrami, P.S.; D’apollonia, S. \& Rosenfield. "The Dimensionality Of Student Ratings of Instruction: What We Know and What We do Not". En R.P. PRRY \& J.C. SMART (Eds.) Effective Teaching in Higher Education: Research and Practice, pp.321-367.New York: Agathon Press. 1997.

Aleamori, L.M. "Student Rating myths versus research facts from 1924 to 1998". En Journal of Personnel Evalation in Education, 13 (2), pp. 153166. 1999.

Álvarez, V.; García, E. Y Flores, J. "Características de la Docencia Mejor Evaluada por los Alumnos en Las Diferentes Áreas de Enseñanza Universitaria". En Revista Española de Pedagogía, № 214, pp. 445-464. 1999.

Arbucle, J.L. Amos User's Guide: Versión 3.6. Chicago, IL: Small Waters. 1997.

Ballantyne, R.; Borthwick, J. \& Packer, J. "Beyond Student Evaluation of Teaching: Indentifying and Addressing Academic Staff Development Needs" . En Assessment \& Evaluation in Higher Education. Vol. 25, 3, pp. 221-236. 2000.

Bentler, P.M. "Multivariate Analysis with Latent Variables: Causal Modeling". En Annual Review of Psychology, 31, pp. 419-456. 1990.
Cajide, J. "Análisis Factorial Confirmatorio de las Características de Calidad Docente Universitario (solución LISREL)". En Bordón, 43 (4), pp. 389-405. 1994.

Centra, J.A. Reflective Faculty Evaluation: Enhancing Teaching and Determining Faculty Effectiveness. San Francisco: Jossey-Bass. 1993.

Cohen, P.A. An update and expanded meta analysis of multisection student rating validity studies. Paper presented at the annual meeting of the American Educational Research Association, San Francisco, CA. 1986.

Cohen, R.J. y Swerdlik, M.E. Psychological Testing And Assessment: An Introduction To Tests And Measurement (4th ed.). Mountain View, CA.: Mayfield. 1999.

Craton, P. y Smith, R.A. "Reconsidering the Unit of Analysis: A Model of Student Ratings of Instruction”. En Journal of Educational Psychology, 82 (2) pp. 207-212. 1990.

De La Orden, A. "Evaluación, Selección y Promoción del Profesor Universitario". En Revista Complutense de Educación. Vol 1 (1), 11-29. 1990.

De Miguel, M. "La Evaluación Del Profesorado Universitario. Criterios y Propuestas Para Mejorar La Función Docente". En Revista de Educación, $\mathrm{N}^{\circ} 315$, pp. 67-83. 1998.

Escudero, T. "Los Estudiantes como Evaluadores de la Docencia y de los Profesores: Nuestra Experiencia”. En Revista Interuniversitaria De Formación Del Profesorado. № 34, Enero/ Abril. Pp. 69-86. 1999. 
Feldman, K.A. "The superior teacher from de students view". En Research in Higher Education, 5, pp. 243288. 1976a.

Feldman, K.A. "Grades and College Student's Evaluation of Their Courses and Teacher". En Research in Higher Education, 18 (1), pp. 3-124. 1976b.

Feldman, K.A. "Seniority and experience of college teachers as related to evaluations they receive from students". Research in Higher Education, 18, 3124. 1983.

Feldman, K.A. "Class size and college students' evaluation of teachers and courses: a closer look". In Research in Higher Education, 21 (11), pp. 45-116. 1984.

Feldman, K.A. "Research Productivity and Scholarly Accomplishment of College Teacher as Related to Their Instructional Effectiveness: A Review and Exploration". En Research in Higher Education, 26 (3), pp. 227298. 1987.

Feldman, K.A. "The association between student ratings of specific instructional dimensions and student achievement: refining and extending the synthesis of data from multisection validity studies". En Research in Higher Education, 30, pp. 583-645. 1989.

Feldman, K.A. "Indentifying Exemplary Teachers and Teaching: Evidence from Student Ratings". En R.P. PERRY \& J.C. SMART (eds.), Effective teaching in Higher Education: Research and Practice, Bronx, N.Y.: Agathon. Pp.368-395. 1997.
Fernández, J.; Mateo, M. \& Muñíz, J. "Is There Relationship Between Class Size and Student Ratings of Teacher Quality?" En Educational And Psychological Measurement, 58 (August), 596-604. 1998.

García Ramos, J. M. y Gálvez Hernádez, M "Un modelo tutorial universitario". En Revista Complutense de Educación, 7 (1), Madrid. Pp. 275303. 1996b.

García Ramos, J.M. "Valoración De La Competencia Docente Del Profesor Universitario: Una Aproximación Empírica”. En Revista Complutense de Educación, 8 (2), Madrid. Pp. 81108. 1997.

García Ramos, J.M. "Análisis Factorial Confirmatorio en la Validación del Constructo Competencia Docente del Profesor Universitaria”. En Bordón, 49 (4), Madrid. Pp. 361-391. 1997b.

García Ramos, J.M. "El Análisis de Estructuras de Covarianza en El Estudio De La Competencia Docente Del Profesor Universitario". En Revista de Investigación Educativa, 16 (1), Madrid. Pp. 155-184. 1998.

Gibbs, G. Improving the Quality of Student Learning. New York: Teacher's College Press. 1992b.

González Such, J.M.; Jornet M., J.M.; Suárez R., J.M. y Pérez C., A. "Análisis de Tipologías de Calidad Docente a Partir de Cuestionario de Evaluación del Profesorado Universitario”. En Bordón, 51 (1), pp. 95-114. 1999.

Green, Sbt.; Akey, T.M.; Fleming, K.K.; Hershberger, S.C. Y Marquis, J.G. 
"Effects of the number of scale points on chi- square fir indices in confirmatory factor analysis". En Structural Equation Modeling, 4 (2), pp. 108120. 1997.

Hair, J.; Anderson, R.; Tatham, Black, W. Análisis Multivariante. España: Prentice Hall. 1999.

Hu, L. \& Bentler, P. M. "Cutoff criteria for fit indexes in covariance structure analysis: Conventional criteria versus new alternatives". En Structural Equation Modeling, 6(1), 1-55. 1999.

Jackson, D.; Teal, C.; Raines, S.; Nansel, T.; Force, R.\& Burdsal Ch. "The Dimensions Of Students' Perceptions Of Teaching Effectiveness". En Educational And Psychological Measurement, vol. 59, (4), pp. 580-596. 1999.

Jornet, J.; Suares, J.M. y González, J. "Proyecto de Implantación de un Sistema de Evaluación del Profesorado en la Universidad de Valencia”. En Consideraciones Metodológicas Sobre La Evaluación Y Mejora De La Docencia Universitaria. Universidad de Valencia, Informes de Investigación Evaluativa, $N^{\circ}$ 1. 1988.

Kolitch, E. \& Dean, A.V. "Student Ratings of Instruction en the U.S.A.: Hidden Assumptions and Missing Conceptions About "Good" Teaching". En Studies in Higher Education, Vol. 24, (1), pp. 27-42. 1999.

Kwan, K.P. "How Fair Are Rating in Assessings The Teaching Performance Of University Teacher?" En Assessment \& Evaluation In Higher Education, 24 (June), 181-195. 1999.

Marsh, H. \& Bailey, M. "Multidimensional Students' Evaluations of Teaching
Effectiveness". En Journal of Higher Education,64 (1), pp. 1-18. 1993.

Marsh, H. \& Roche, L. R. "Making Students' Evaluation of Teaching Effectiveness Effective: The Critical Issues of Validity, Bias, and Utility". En American Psychologist, Vol. 52, 11, 1187-1197. 1997.

Marsh, H. "SEEQ: a Realiable, Valid, and Useful Instrument for Collecting Students Evaluation of University Teaching". En British Journal of Psychology, 52, pp. 77-95. 1982.

Marsh, H. "Student's evaluation of university teaching; dimensionality, reliability, validity, potential biases ad utility". En Journal of Educational Psychology, 76 (5), 707-754. 1984.

Marsh, H. W., \& Dunkin, M. J. 'Students' evaluations of university teaching: A multidimensional perspective". En J.C. SMART (ed.) Higher Education: Handbook of Theory and Research, (Vol. 8, pp. 143-233). New York: Agathon Press. 1992.

Marsh, H.W. "Student's Evaluation of Tertiary Instruction: Testing the Applicability of American Surveys in an Australian Setting". En Australian Journal of Education, 25, pp. 177-192. 1981.

Marsh, H.W. y Roche, L. The Use of Student's Evaluations of University Teaching To Improve Teaching Effectiveness. Canberra: Australian Government Publishing Services. 1994.

Marsh, H.W. y Roche, L.A. "The Use of Student Evaluations of University Teaching in Different Settings: The 
Applicability Paradigm". En Australian Journal of Education, 36 (3), pp. 278-300. 1992.

Marsh, H.W. y Roche, L.A. "Effects of grading leniency and low workload on students' evaluation of teaching: popular myth, bias, validity or innocent bystanders?" En Journal of Educational Psychology, 92 (1), pp. 202-228. 2000.

Marsh, H.W.; Touron, J. y Wheeler, B. "Students' s Evaluations of University Instructor: The Applicability of American Instrument in a Spanish Setting”. En Teaching and Teacher Education, 1, pp. 123-138. 1985.

Mckeachie, W.J. "Research on College Teaching: The Historical Background". En Journal of Educational Psychology, 82 (2), pp. 189-200. 1990.

Murray, H.G. "Does Evaluation of Teaching Lead to Improvement of Teaching?" En International Journal for Academic Development, 2 (1), pp. 2041. 1997.

Patrick, J. y Smart, R.M. "An Empirical Evaluation of Teacher Effectiveness: The Emergence of Three Critical Factors". En Assessment and Evaluation in Higher Education. 23 (2) pp. 165-178. 1998.

Prosser, M. \& Trigwell, K. "How will Future Academic be Evaluated? Using Student Study Strategies to Check the Validity of Student Evaluations of Teaching Courses". En G. MULLIS (Ed) Breaking the Mould. Paper Presented at the Fifteenth Annual
Conference of HERDSA, pp. 282.284. Sydney: HERDSA. 1990.

Ramsden, P. Learning of Teach in Higher Education. London: Routledge. 1992.

Rindermann, H. \& Schofield, N. "Generalizability of Multidimensional Student Rating of University Instruction Across Courses and Teacher". En Research in Higher Education. Vol. 42, 4, pp. 377-400. 2001.

Schalock, M.D. “Teacher Productivity Revised: Definition, Theory, Measurement And Aplication". En Journal Or Personnel Evaluation In Education, 8 (2), pp. 179-196. 1993.

Swartz, C.W.; White, K.P. y Stuck, G.B. "The Factorial Structure of The North Caroline Teacher Performance Appraisal Instrument”. En Educational and Psychological Measurement, 50 (1), pp. 175-185. 1990.

Tejedor, F. y Montero, L. "Indicadores de la Calidad Docente para La Evaluación del Profesor Universitario". En Revista Española de Pedagogía, año XLVIII, $\mathrm{N}^{\circ} 186$, mayo-agosto, pp. 259-279. 1990.

Ting, K.F. "A Multilevel Perspective On Student Ratings of Instruction: Lessons From the Chinese Experience". En Research in Higher Education. Vol. 41, 5, pp. 637-653. 2001.

Young, P.; Delli, D. y Johnson, L. "Student Evaluation of faculty: Effects of Purpose on Pattern". En Journal of Personnel Evaluation in Education, 13 (2), pp. 179-190. 1999. 


\begin{tabular}{|c|c|}
\hline Indicadores & Items \\
\hline Preparación de clases & $\begin{array}{l}\text { 1- Evidencia que ha preparado las clases con anticipación. } \\
\text { 4- Los objetivos de curso se definen anticipadamente. } \\
\text { 3- Realiza un resumen del tema anterior, antes de iniciar } \\
\text { cada clase. } \\
\text { 9- Cumple con el desarrollo de los temas de acuerdo con el } \\
\text { programa del curso. }\end{array}$ \\
\hline $\begin{array}{l}\text { Utilización de m\#todos } \\
\text { y recursos de instrucción }\end{array}$ & $\begin{array}{l}\text { 10- Emplea variedad de ayudas audiovisuales (multimedia, } \\
\text { diapositivas, v"deos, etc). } \\
\text { 13- Utiliza variedad de recursos de ense\%anza (demostraciones, } \\
\text { lecturas, trabajo en grupo, etc). }\end{array}$ \\
\hline Dominio de los temas & 12- Muestra dominio de los temas tratados. \\
\hline Exposición (claridad y orden) & $\begin{array}{l}\text { 14- Es ordenado al exponer los contenidos de la clase. } \\
\text { 16- Explica con claridad. } \\
\text { 18- Resume y enfatiza los aspectos claves de cada lección. }\end{array}$ \\
\hline Amplitud & $\begin{array}{l}\text { 7- Contrasta varias implicaciones en los temas que explica. } \\
\text { 8- Brinda un contexto global a sus ideas y conceptos. }\end{array}$ \\
\hline $\begin{array}{l}\text { Justicia e imparcialida } \\
\text { a la hora de evaluar y calificar }\end{array}$ & $\begin{array}{l}\text { 19- El o los procedimientos de evaluación permiten } \\
\text { al alumno reflejar sus conocimientos. } \\
\text { 22- Elabora evaluaciones (ex! menes, quices, tareas, } \\
\text { asignaciones, otros) para sintetizar efectivamente la } \\
\text { materia del curso. } \\
\text { 24- Es justo e imparcial a la hora de evaluar y calificar } \\
\text { (ex! menes, quices, tareas, asignaciones, otros). } \\
\text { 26- Devuelve las evaluaciones ya calificadas dentro de los diez } \\
\text { d"as h! biles siguientes a su realización o entrega. } \\
\text { 28- Genera una retroalimentación en los ex! menes, } \\
\text { calificaciones y materiales, que me ayudan comprender } \\
\text { aún m! s la materia de clase. }\end{array}$ \\
\hline $\begin{array}{l}\text { Dificultad, cantidad de trabajo } \\
\text { Expectativa e inter\#s } \\
\text { Retroalimentación de la } \\
\text { valoración de clase }\end{array}$ & $\begin{array}{l}\text { 29- Comparado con otros, la dificultad de este curso fue } \\
\text { 30- Relacionado con otros, la cantidad de trabajo que se } \\
\text { asigna en este curso es } \\
\text { 31- Tengo expectativas de obtener una nota de } \\
\text { 32- Has aprendido en este curso, algo que consideras } \\
\text { de valor para tu desarrollo profesional o humano. }\end{array}$ \\
\hline $\begin{array}{l}\text { Estimula y provoca la } \\
\text { participación, el diálogo, } \\
\text { la reflexión y debate. } \\
\text { Escucha dudas y críticas }\end{array}$ & $\begin{array}{l}\text { 2- Brinda la oportunidad de exponer dudas y preguntas, } \\
\text { respondi\$ndolas con claridad. } \\
\text { 5- Acepta cr"ticas a sus objetivos de clase, planteamientos } \\
\text { y otros, por parte de los estudiantes y las estudiantes. } \\
\text { 6- Se interesa por los estudiantes y las estudiantes que } \\
\text { demuestranproblemas en aprender, los temas de estudio, } \\
\text { al mismo ritmo que la clase. } \\
\text { 17- Mantiene buenas relaciones con los estudiantes. }\end{array}$ \\
\hline
\end{tabular}




\begin{tabular}{|c|c|}
\hline Apoya a los estudiantes & $\begin{array}{l}\text { 11- Se encuentra disponible en el horario que estableció } \\
\text { para horas de consulta extra clase. } \\
\text { 15- Trata de acercarse y conversar con los estudiantes } \\
\text { antes y después de clase. }\end{array}$ \\
\hline $\begin{array}{l}\text { Entusiasmo e interás } \\
\text { mostrado en clase }\end{array}$ & $\begin{array}{l}\text { QBfleja entusiasmo en la presentaci ón de la clase } \\
\text { o de los materiales. } \\
\text { \&- Tiene una forma de ense í ar que facilita la comprensión } \\
\text { de la materia }\end{array}$ \\
\hline Actitud activa y din\#mica & $\begin{array}{l}\text { 8Cuando explica mantiene mi atenci ón en la clase. } \\
\text { 8- Se mantiene activo y din \#mico cuando dirige este curso } \\
\text { Z Aiste regularmente a clases y si falta lo justifica. }\end{array}$ \\
\hline Ítem Criterios & $\begin{array}{l}\text { 3Considera usted que este curso ha sido } \\
\text { 3En general usted dir !a que su profesor es }\end{array}$ \\
\hline
\end{tabular}

